\title{
A probable case of sodium valproate monotherapy induced drug reaction with eosinophilia and systemic symptoms syndrome: a case report in Indian population
}

\author{
Vijayamathy Arunnair ${ }^{1 *}$, Girish Kumaraswamy ${ }^{2}$, Ramesh M. ${ }^{3}$
}

\begin{abstract}
${ }^{1}$ Department of Pharmacology, KMCH Institute of Health Sciences, Coimbatore, Tamilnadu, India
${ }^{2}$ Department of Pharmacology, ${ }^{3}$ Department of Dermatology, Kempegowda Institute of Medical Sciences, Bangalore, Karnataka, India
\end{abstract}

Received: 12 April 2020

Revised: 09 May 2020

Accepted: 11 May 2020

\section{*Correspondence:}

Dr. Vijayamathy Arunnair,

Email: vijiabhi75@gmail.com

Copyright: (C) the author(s), publisher and licensee Medip Academy. This is an open-access article distributed under the terms of the Creative Commons Attribution Non-Commercial License, which permits unrestricted non-commercial use, distribution, and reproduction in any medium, provided the original work is properly cited.

\begin{abstract}
Drug reaction with eosinophilia and systemic symptoms (DRESS) syndrome is a drug-induced hypersensitivity syndrome most commonly caused by anti-epileptics like carbamazepine, phenytoin. It usually manifests as maculopapular rash, fever, eosinophilia, generalized lymphadenopathy, hepatitis, atypical lymphocytosis, and leucocytosis with the involvement of other organs. Valproate, a broad-spectrum antiepileptic, also used in bipolar disorders and migraine prophylaxis, is generally well tolerated. To date, very few cases of valproate monotherapy induced DRESS syndrome have been reported worldwide and here we are reporting one such rare case in a 72 years old Indian woman with a history of generalised tonic convulsions. The patient was classified as a 'probable' case of DRESS syndrome using the WHO-UMC criteria and Naranjo scale. Discontinuation of the offending medication and treatment with intravenous (IV) fluids, antihistamines, and steroids helped in recovery of the patient. Healthcare practitioners must be aware of valproate monotherapy induced DRESS syndrome and take effective measures to avoid severe side effects.
\end{abstract}

Keywords: Valproate, DRESS, Adverse drug reaction, Anti-epileptic drugs

\section{INTRODUCTION}

The syndrome of drug reaction with eosinophilia and systemic symptoms (DRESS) also known as druginduced hypersensitivity syndrome (DIHS), is a potentially lethal type of severe cutaneous adverse reactions (SCARs) with an incidence of 1 in 1000 to 1 in 10,000 drug exposures and a mortality rate of around 10 to $20 \% .^{1-4}$ It is characterized by generalized acute maculopapular eruptions with high-grade fever and multivisceral involvement. ${ }^{1}$ Generally, there is a late onset of symptoms (3 weeks to 3 months) after the start of the offending drug which may persist or worsen despite the withdrawal of the offending drug. ${ }^{3}$ The most common offending drugs are anti-epileptic drugs (AEDs) (carbamazepine, phenytoin, phenobarbitone, lamotrigine, zonisamide, oxcarbazepine), allopurinol, sulphonamide, dapsone, minocycline, nevirapine. ${ }^{4}$ Non-aromatic AEDs such as valproate, gabapentin, vigabatrin, levetiracetam were traditionally thought to be safer. ${ }^{5}$ DRESS syndrome is diagnosed by RSCAR (the registry of severe cutaneous adverse drug reactions) criteria which encompass the following: an acute rash, lymphadenopathy at two sites, fever above $38^{\circ} \mathrm{C}$, the involvement of at least one internal organ and abnormality of lymphocyte and eosinophil counts. $^{6-8}$ Valproate which is one of the frontline antiseizure drug used in multiple types of seizures and the 
side effects of valproate are many and well known which includes gastrointestinal upset, sedation, ataxia, and tremor. $^{2}$ However, serious adverse reactions such as DRESS, hepatotoxicity, encephalopathy, coagulation disorders, pancreatitis, and bone marrow suppression are rare. ${ }^{5}$ As there are few case reports in Indian literature to evaluate the causality of a rare case of valproate induced DRESS syndrome and hence the present report throws light on the importance of recognizing the possibility of SCARs associated with valproate.

\section{CASE REPORT}

Valproate induced DRESS syndrome was seen in a 72 years old female, who had a history of generalized tonic convulsion two months back and she was prescribed sodium valproate $500 \mathrm{mg}$ twice a day. After one month she had developed a generalized skin rash, scaling of skin over the scalp, face, trunk, and both upper and lower limbs, crusting, facial edema, high-grade fever which was on and off, more in the night and associated with chills and rigor, for which she got admitted in a tertiary care hospital in Bangalore, after 3 days of illness. On general examination, the patient's vitals were stable, with blood pressure $100 / 70 \mathrm{mmHg}$ and pulse rate $68 \mathrm{bpm}$. Laboratory findings were leucocytosis $(11,070$ cells $\left./ \mathrm{mm}^{3}\right)$, eosinophilia $(\mathrm{E}=43.4 \%, \mathrm{AEC}=4800$ cells $)$, elevation of liver enzymes $($ SGOT=354 IU/1, SGPT=287 IU/l). Other investigations like dengue profile, malarial parasite, widal, platelet count, urine culture, random blood sugar, renal function tests were negative or within normal limits. Based on the RegiSCAR scoring system the patient had a score of 5 points, which indicated a probable case of valproate induced DRESS syndrome. The offending drug (valproate) was discontinued and levetiracetam $500 \mathrm{mg}$ twice a day was substituted. Subsequently, the patient was managed with IV fluids, antihistamines, and parenteral betamethasone once daily for 5 days and it was replaced by tapering doses of oral prednisolone $10 \mathrm{mg}, 5 \mathrm{mg}$ once daily for two weeks. Symptoms resolved and laboratory parameters returned to normal levels after 8 days. No new episode of seizure was reported and she showed complete recovery at the time of discharge (10 days).

\section{DISCUSSION}

DRESS syndrome is a hypersensitivity reaction to drugs, with a delayed onset. In our case it was one month after taking the offending drug, similar to other reports and it also produces fatal organ failures (especially liver and lungs) leading to a higher rate of mortality. ${ }^{1,2}$ Aromatic anticonvulsants like carbamazepine, phenytoin, and lamotrigine have been the culprit drugs. ${ }^{1,2}$ Though nonaromatic AEDS like valproate were thought to be safer; there is $70 \%$ cross-reactivity between aromatic AEDs and non-aromatic AEDs. ${ }^{9}$

Various mechanisms postulated include the presence of circulating antibodies or toxic metabolites and reactivation of HHV 6 infection. ${ }^{4}$ It is suggested that aromatic AEDs produce DRESS since they are metabolized by CYP450 to an arene oxide metabolite which is detoxified by epoxide hydrolase enzyme, which may be deficient/mutated genetically in some individuals. ${ }^{4}$ In our case, the safer, non-aromatic AED, valproate (branched-chain fatty acid) is mainly metabolized by oxidation (CYP2C9, 2C19) and by hepatic glucuronidation has initiated DRESS and the exact mechanism is unclear, though genetic predisposition may play a role in this SCAR..$^{1-5}$

Criteria for the diagnosis have been given by the Japanese study group of severe cutaneous adverse reactions to drugs (SCAR-J) and the European group, RegiSCAR. ${ }^{6-8}$ In our case, probable features of valproate induced DRESS were present (score of 5 on RegiSCAR) (Table 1) ${ }^{6-8}$ Valproate was stopped and levetiracetam, systemic steroids were prescribed. Though systemic corticosteroids are warranted only in the severe form of DRESS, most physicians prescribe it even in mild forms of DRESS, because of the higher propensity of relapse, similar to a study done by Brentano et al. ${ }^{10}$ Identical to our case, a 73 years old male with valproate induced DRESS, was managed by the withdrawal of the offending drug and replacing it with levetiracetam and successfully treated with pulse methylprednisolone followed by oral prednisone. ${ }^{1}$ Similar line of management was also followed in a 12 years old boy with valproate induced DRESS. ${ }^{11}$ Even intravenous immunoglobulin, cyclosporine has been tried in severe cases. ${ }^{1,2}$

Table 1: RegiSCAR scoring*.

\begin{tabular}{|l|l|}
\hline Clinical and laboratory criteria & $\begin{array}{l}\text { RegiSCAR } \\
\text { scoring }\end{array}$ \\
\hline Fever $\mathbf{> 3 8 . 5}^{\circ} \mathbf{C}$ & 0 (yes) \\
\hline Enlarged lymph nodes & 0 \\
\hline Eosinophilia & 2 \\
\hline Atypical lymphocytes & 0 \\
\hline Skin rash $>\mathbf{5 0 \%}$ of BSA & 1 \\
\hline Skin rash suggesting DRESS & 1 \\
\hline Biopsy suggesting DRESS & 0 (unknown) \\
\hline Organ involvement & 1 (liver) \\
\hline Resolution $>\mathbf{1 5}$ days & 0 \\
\hline $\begin{array}{l}\text { ANA, blood culture, serology } \\
\text { for HAV/HBV/HCV, } \\
\text { chlamydia, mycoplasma }\end{array}$ & 0 (No) \\
\hline Total & 5 \\
\hline RSCAR & Probable \\
\hline
\end{tabular}

*BSA: body surface area, ANA: anti-nuclear antibody, HAV: hepatitis A virus, HBV: hepatitis B virus, HCV: hepatitis C virus, Final score <2- no case, 2-3- possible case, 4-5- probable case, and $>5$ - definite case.

The causality assessment for the adverse drug reaction (ADR) according to the Naranjo scale which is a 10 items questionnaire, the score was 5 , and so it was classified as a 'probable' case of valproate induced DRESS, so also 
based on WHO-UMC criteria. ${ }^{12,13}$ Severity assessment as per modified Hartwig and Siegel scale, where ADRs are classified into seven levels based on the severity and our case falls into level $4 \mathrm{~b}$ - moderate severity. ${ }^{14}$ As per modified Schumock and Thornton scale, (9 items questionnaire) it was 'probably preventable' (Table 2). ${ }^{15}$

Table 2: ADR assessment.

\begin{tabular}{|ll|}
\hline Scales & ADR assessment \\
\hline WHO-UMC causality & Probable \\
\hline Naranjo's probability & Probable \\
\hline $\begin{array}{l}\text { Modified Hartwig and Siegel } \\
\text { severity }\end{array}$ & Moderate \\
\hline $\begin{array}{l}\text { Modified Schumock } \\
\text { Thornton preventability }\end{array}$ & Probably preventable \\
\hline
\end{tabular}

There are few case reports of DRESS with multiorgan dysfunction due to the combination of valproate with lamotrigine or carbamazepine, not as valproate monotherapy. DRESS syndrome due to concomitant administration of valproate and lamotrigine/ carbamazepine was described by Nakhi et al and Lorido et al respectively. ${ }^{16,17}$ The possible explanation could be multiple drug hypersensitivity as proposed by Pichler et al due to substitution therapy of valproate (as a second drug) with other aromatic AEDs causing activation of the immune system, thereby leading on to DRESS. ${ }^{18}$ This fact is endorsed by a case of a 25 years old woman with bipolar disorder who experienced valproate induced DRESS one month after lamotrigine withdrawal due to nonspecific rash. ${ }^{1}$

A small number of cases of DRESS syndrome were reported, when valproate was combined with other drugs apart from AEDs and in the presence of other comorbidities. According to an Indian study, DRESS syndrome was encountered in a 39 years old male diagnosed with a recurrent depressive disorder who was under treatment with escitalopram and sodium valproate. ${ }^{3}$ Another case of DRESS was reported in a 20 years old Brazilian female who took valproate and haloperidol. ${ }^{19}$ An adolescent with symptoms of mania, developed DRESS syndrome when valproate was combined with olanzapine. ${ }^{20}$ A 60 years old Iranian male developed DRESS syndrome with prophylactic valproate (1000 $\mathrm{mg}$ /day) therapy, after subarachnoid haemorrhage and concomitant insulin therapy for diabetes. ${ }^{2}$ A 19 years old female, a case of brucellosis, who was under treatment with valproate (chorea), doxycycline capsules and streptomycin as polytherapy developed DRESS. ${ }^{21}$ In this case probably the leading cause might be brucellosis, though not known. A 73 years old Chinese male, a case of atrial fibrillation, on treatment with warfarin suffered DRESS with other complications like hyponatremia, thrombocytopenia, hypoalbuminemia, high aminotransferase when he was put on $1000 \mathrm{mg} /$ day valproate for seizures. ${ }^{1}$ DRESS with acute liver failure was reported in a 26 years old man with valproate, clemastine, baclofen, and acetaminophen. ${ }^{22}$ Though our report was a rare case of valproate monotherapy induced DRESS syndrome without any precipitating/concomitant factors/drug combinations in an adult female, future studies should direct towards identifying the risk factors for DRESS, which may be due to valproate monotherapy or polytherapy or existing comorbidities.

Therefore, it was a probable case of valproate monotherapy induced DRESS syndrome with moderate severity and probably preventable. The health care professionals (HCPs) need to keep in mind about the risk of DRESS syndrome even with non-aromatic AED like valproate as a monotherapy, (no concomitant illness/multidrug) as well as the cross-reactivity between aromatic anticonvulsants and valproate. Switching the patient to different safer drugs like levetiracetam and treating with systemic corticosteroids may prove to be useful, similar to our case.

\section{CONCLUSION}

To the best of our knowledge, very few cases of valproate monotherapy induced DRESS was reported in Indian population. Valproate being the frontline AED, proper subject/public instructions, by the HCPs regarding the offending drugs that cause hypersensitivity reactions like DRESS, prompt early reporting, and recognition of this cutaneous ADRs with timely initiation of therapy could prevent or minimize the involvement of other organs/ mortality and also aid in pharmacovigilance activities, so as to propose future guidelines for safer prescribing. Though aromatic AEDs are proposed to be safer, based on our report, still there is an unmet need to explore the mechanisms involved in valproate monotherapy induced DRESS syndrome. A vigilant attitude towards multidrug hypersensitivity with valproate substitution therapy is pivotal.

\section{Funding: No funding sources \\ Conflict of interest: None declared \\ Ethical approval: Not required}

\section{REFERENCES}

1. Wu XT, Hong PW, Suolang DJ, Zhou D, Stefan H. Drug-induced hypersensitivity syndrome caused by valproic acid as a monotherapy for epilepsy: First case report in Asian population. Epilepsy Behav Case Rep. 2017;8:108-10.

2. Darban M, Bagheri B. Drug Reaction with Eosinophilia and Systemic Symptoms induced by Valproic Acid: A Case Report. Iran Red Crescent Med J. 2016;18(9):36825.

3. Dwivedi AK, Ray M, Raj J, Saini RK. Valproate induced drug reaction with eosinophilia and systemic symptoms (DRESS). Indian J Psychiatry. 2018;60:512-3.

4. Cacoub P, Musette P, Descamps V, Meyer O, Speirs C, Finzi L, et al. The DRESS Syndrome: A Literature Review. Amer J Med. 2011;124:588-97. 
5. Primec RZ, Balkove V. Valproate-related erythrodermia with reversible encephalopathy: a rare but serious adverse reaction, case report. Acta Dermatovenerol APA. 2014;23:35-7.

6. Kardaun SH, Sidoroff A, Allanore VL, Halevy S, Davidovici BB, Mockenhaupt M, et al. Variability in the clinical pattern of cutaneous side-effects of drugs with systemic symptoms: Does a DRESS syndrome really exist. Br J Dermatol. 2007;156:609-11.

7. Eshki M, Uanore AL, Musette P. Twelve years analysis of severe cases of drug reaction with eosinophilia and systemic symptoms: a cause of unpredictable multiorgan failure. Arch Dermatol. 2009; 145:67-72.

8. Choudhary S, Leod M, Torchia D, Romanelli P. Drug Reaction with Eosinophilia and Systemic Symptoms (DRESS) Syndrome. J Clin Aesthet Dermatol. 2013;6(6):31-7.

9. Meik S, Arias M, Fernandez L, Santoro LMC, Abeldano A, Pellerano G. Anti-convulsant hypersensitivity syndrome (DRESS syndrome): report of 4 cases. Dermatol Argent. 2010;16(4):272-7.

10. Brentano FE, Duong TA, Bouvresse S, Bagot M, Wolkenstein P, Roujeau JC, et al. Therapeutic management of DRESS: a retrospective study of 38 cases. J Am Acad Dermatol. 2015;72(2):246-52.

11. Kocaoglu C, Cilasun C, Solak ES, Kurtipek GS, Arslan S. Successful Treatment of Anti-epileptic Drug-Induced DRESS Syndrome with Pulse Methylprednisolone. Case Rep Pediatr. 2013;2013:1-4.

12. Naranjo CA, Busto U, Sellers EM, Sandor P, Ruis I, Roberts EA, et al. A method for estimating the probability of adverse drug reactions. Clin Pharmacol Ther. 1981;30:239-45.

13. The use of the WHO-UMC system for standardized case causality assessment. Available at: http://www. WHO-UMC.org/graphics/4409.pdf. Accessed on 12 February 2011.

14. Hartwig SC, Siegel J, Schneider PJ. Preventability and severity assessment in reporting adverse drug reactions. Am J Hosp Pharm. 1992;49:2229-32.
15. Schumock GT, Thornton JP. Focusing on the preventability of adverse drug reactions. Hosp Pharm. 1992;27:538.

16. Nakhi BHA, Sadeq S, Pinto RG, Habeeb $Y$. Anticonvulsant hypersensitivity syndrome: report of 2 cases from Kuwait. Med Princ Pract. 2003;12(3):197-9.

17. Lorido AJC, Gomez CJ, Dacal BJC, Leal MC, Dacal BP. Anti-epileptic drug hypersensitivity syndrome in a patient treated with valproate. Br J Clin Pharmacol. 2003;55(4):415-6.

18. Pichler WJ, Srinoulprasert Y, Yun J, Hausmann O. Multiple drug hypersensitivity. Int Arch Allergy Immunol. 2017;172(3):129-38.

19. Silva SA, Figueiredo MM, Carneiro LN, Reiss DB, Damasio MA. Drug reaction with eosinophilia and systemic symptoms (DRESS syndrome). Rev Assoc Med Bras. 2016;62(3):227-30.

20. Penchilaya V. DRESS syndrome: Addressing the drug hypersensitivity syndrome on combination of Sodium Valproate and Olanzapine. Asian J Psychiatr. 2017;28:175-6.

21. Albayrak F, Cerrah S, Albayrak A, Dursun H, Yildirim R, Uyanik A. DRESS syndrome with fatal results induced by sodium valproate in a patient with brucellosis and a positive cytoplasmic antineutrophilic cytoplasmic antibody test result. Rheumatol Int. 2012;32(7):2181-4.

22. Zoelan VMA. Valproic acid induced DRESS syndrome with acute liver failure. Neth $\mathbf{J}$ Med. 2012;70(3):155.

Cite this article as: Arunnair V, Kumaraswamy G, Ramesh M. A probable case of sodium valproate monotherapy induced drug reaction with eosinophilia and systemic symptoms syndrome: a case report in Indian population. Int J Basic Clin Pharmacol 2020;9:991-4. 